\title{
Knowledge, Attitude, and Practices Regarding Nutrition for Patients With Chemotherapy
}

\author{
Neama Fosdok William ${ }^{1}$, Mimi Mohamed Mekkawy ${ }^{2}$, Sahra Zaki Azer ${ }^{3}$, Medhat A. Saleh ${ }^{4} \&$ Salah Mabrouk \\ Khallaf ${ }^{5 .}$ \\ 1- B.SC. Nursing Science Faculty of Nursing in South Egypt Cancer Institute Assiut, University, Egypt. \\ 2 - Professor of Medical Surgical Nursing Faculty of Nursing, Assiut University Egypt. \\ 3- Assistant Professor of Medical Surgical Nursing Faculty of Nursing, Assiut University, Egypt. \\ 4- - Assistant Prof of Public Health and Community Medicine, Faculty of Medicine, Assiut Uinversity, Egypt. \\ 5- Lecturer of Medical Oncology and Hematological Oncology at South Egypt Cancer Institute, Egypt.
}

\begin{abstract}
Background; good nutrition \& eating are important especially during treatment which might enhance the effectiveness of tumor treatments \& which can lead to better outcomes for cancer treatment. The aim: assess Knowledge, attitude, and practices regarding nutrition for patients with chemotherapy. Samples: A convenience sample of 60 adult patients, the age between 20 - 60 years both sex, evidence of cancer and recent receiving all types of chemotherapy. Three tools were utilized for data collection were Tool (I) Structured interviewing Questionnaire Tool (II) Nutritional Status Assessment and Tool (III) Chemotherapy Knowledge, Attitude and practice (KAP) Questionnaire. Results: More than two thirds of samples were unsatisfied level of knowledge (60\%). The majority of studied sample were positive attitude and poor practice $(95 \%, 86.6 \%)$ respectively. Conclusions: About two third of the patient were unsatisfactory information. The majority of the patient were positive attitude and had poor practice respectively. Recommendations: Give advices \& guidelines for patients to minimize malnutrition complications \& improve clinical outcomes, formulate nutritional assessment form or sheet to be suitable for Egyptian patients undergoing chemotherapy in the hospitals.
\end{abstract}

\section{Keywords: Attitude, Chemotherapy, Knowledge \& Practice.}

\section{Introduction}

Chemotherapy is the cancer treatment most likely to weaken the immune system. Chemotherapy medicines target rapidly dividing cells, in blood, bone marrow, mouth, intestinal tract, nose, nails, vagina, and hair. Cancer cells are destroyed by chemotherapy because they can't repair themselves very well, the healthy cells typically can repair the damage from chemotherapy once treatment ends. (Jemal et al., 2011).

Beneficial nutrition is important, good eating during medication can improve effect of treatments on tumor, which can prompt better results for cancer treatment (Morello et al., 2010).

Cancer treatment may affect one's diet, and dietary changes can exacerbate other treatment related symptoms. For example, cancer patients who experience chemosensory alterations, distorted taste, and increased sensitivity to smells due to the cancer itself or as a side effect of treatment are higher levels about weight loss, lower energy intake, \& quality of life is worse. Aprisingly high number of patients undergoing cancer treatment report distortions to their sense of taste or smell. Cancer patients also report early satiety and food aversions as a result of treatment. Dietary interventions, such as dietary counseling, flavor enhancement, oral supplementation, or tube feeding, have been found to lessen weight loss and improve health status (Mariani \& Bozzetti., 2012)

The dietary approach might be helping to raise cancer treatment to a new level of success as supplementing or/and supporting those figure for regular phytochemicals can not decrease side effects but enhance also the adequacy for chemotherapeutics. Different plant-derived exacerbates enhance those effectiveness from claiming cytotoxic agents, decline their resistance, bring down furthermore allay dangerous side effects, diminish those danger from claiming tumor lysis syndrome. Also detoxify the body of chemotherapeutics. The customize approach utilizing different phytochemicals gives another measurement of the standard cancer therapy for help to enhancing its outcome previously, an intricate and integral route (Koyabu \& Ohtain, 2012).

\section{Significance of the study}

Nutrition is a vital role for diminishing morbidity \& mortality in patients undergoing chemotherapy. According to South Egypt cancer institute foundation records 2014-2015 it has been discovered that number of patients admitted to the hospital (5338)

(South Egypt Cancer Institute (diagnosed with cancer and received chemotherapy. From the researcher's clinical experience it was observe that all patients ask more questions about types, amounts of nutrition, and 
misunderstanding about malnutrition. This was the first study to provide assessment knowledge, attitude, and practices for patients with chemotherapy

\section{Aim of the study}

To assess knowledge, attitude, and practices regarding nutrition for patients with chemotherapy.

\section{Research question}

Is there relation between knowledge, attitude, and practices about nutrition for patients undergoing chemotherapy?

\section{Patients \& Method}

Research design:

Descriptive research design was utilized.

\section{Setting}

The study was conducted in oncology (medical and surgical) department at South Egypt Cancer Institute at Assiut University.

\section{Patients}

A convenience sample of 60 adult patients, the age between 20 - 60 years both sex, evidence of cancer and recent receiving all types of chemotherapy

Tools

Three tools were utilized to collect data for this study:

Tool one: Structured interviewing Questionnaire

To assess data and medical history for patients undergoing chemotherapy. Was chemotherapy. The researcher was developed this tool after reviewing, utilizing the most recent \& relevant literature, it comprised of three parts:

Part one: Demographic data: e.g., patient sex, age, occupation, level about education, marital status, residence, \& occupation.

Part two: Disease history and laboratory investigations: such as family history about cancer, span of illness, type, stage, and site of cancer. Types and response to chemotherapy and laboratory investigations

\section{Part three: Dietary habits}

Part four: performance status The Eastern Cooperative Oncology Group (ECOG):

Tool two: Assessment of Nutritional Status:

To assess status of nutrition for patient. It included two parts.

Part one: Anthrometric measurement: It included assessing weight (in $\mathrm{kg}$ ), height \& body mass index $(\mathrm{BMI}))=$ weight $($ in $\mathrm{kg}) /$ Height $(\text { in meter })^{2}$

- Under weight $<20$

- Standard level $20<26$

- Over weight $26<30$

- Obese $30<40$

- Morbid obesity >40
This equation and classification of BMI were adopted from (Jcoyle, 2013)

Tool three: Chemotherapy Knowledge -Attitude practice (KAP) Questionnaire

It was developed on the basis of (Nafissi \&

Raychaudhuri, 2012). Consist of three dimensions and contains 25 items (knowledge dimensions $=14$ items, attitude dimensions $=5$ items and practice dimensions $=6$ items).

Scoring system

Each complete answer get a score of (2) and incomplete answer was get a score of (1) but unknown answer get a score of (0). Patient who scored $(70 \%)$ or more were considered as having "very good" knowledge. Those who scored (60 > $70 \%)$ were considered having "good" knowledge while those scored $(50>60 \%)$ were considered as having "fair" knowledge. Less than (50\%) were considered poor.

Patients were also asked to indicate on three.Likert scale their agreement about attitude where $0=$ disagree, $2=$ agree, $1=$ neutral. The total score of the scale was obtained by summing up responses for 5 , the range from $0-10$ agree $(<50 \%)$, neutral agree $(50-$ $75 \%) \&$ highly agree $(>75 \%)$

The observation (practice was scored in the form of done $=2$ degree, not done $=1$ degree, unknown $\&$ not applicable $=0$ degree. Accordingly, the overall score of observation checklist ranging from $0-12$.

Methods

- Content validity might have been finished by 5 expertise from staff of nursing \& medical, adjustments were made accordingly, and tools were designed in their final format

- An official consent might have been gotten from the head of the South Egypt cancer institute at Assiut University after explaining the aim of study to conduct the study.

Ethical considerations

- Research proposal was might have been affirmed from ethical committee in the faculty of nursing

- There is no hazard to study patients throughout research application.

- An oral consent might have been acquired starting with patients to participate in the study and purpose of the study must be explained to them.

- The researcher at first acquainted herself will every one patients and they were assured that the gathered information might a chance to be absolutely private.

- They were informed the patients may be voluntary and they might withdraw at any time of the study.

- Confidentiality of the patient's information might have been determined. 
- Patient's names were coded for information entry so that their names might not a chance to be distinguished.

Pilot study: might have been directed on $10 \%$ of the patient (6) to assess the applicability \& clarity of the tools. As stated by this pilot study, those required adjustments were aggravated. Patients who were included in the pilot study were excluded from the study.

Content reliability: The tools were designed in their final format and tested for reliability using internal consistency for all of the tools which might have been measured utilizing Cronbach test. Those tools demonstrated will be dependable at $(0.73 .0 .71 \&$ 0.81 , respectively).

\section{Statistical design}

Data entry might have been carried out utilizing perfect individual check by those analysts. Every one information might have been entered under measurable bundles for those social sciences (SPSS) rendition 17. 0 software for analysis and Excel for figures. The content of each tool might have been analyzed, categorized and then coded by the researcher. Information were exhibited utilizing descriptive statistics in the form of frequencies \& percentages for qualitative variables, and means and standard deviations for quantitative variables. Association Investigation might have been utilized to appraisal of the inter-relationships around quantitative variables. Statistical significance difference was considered when statistical significance was considered at p-value.

\section{Results}

Table (1): Distribution of demographic characteristics of patients $(n=60)$.

\begin{tabular}{|c|c|c|}
\hline Items & N. & $\%$ \\
\hline \multicolumn{3}{|l|}{ 1-Age } \\
\hline • $18-29$ yrs. & 3 & 5 \\
\hline - $\quad 30-40$ yrs & 18 & 30 \\
\hline - $41-49$ yrs & 22 & 36.7 \\
\hline - $50-65$ yrs & 17 & 28.3 \\
\hline \multicolumn{3}{|l|}{2 -Sex: } \\
\hline - $\quad$ Patientts & 60 & 100 \\
\hline \multicolumn{3}{|l|}{ 3- Marital status: } \\
\hline$\bullet \quad$ Single & 1 & 1.7 \\
\hline - $\quad$ Married & 58 & 96.7 \\
\hline - $\quad$ Divorced & 0 & 0 \\
\hline - $\quad$ Widowed & 1 & 1.7 \\
\hline \multicolumn{3}{|l|}{ 4-Education level: } \\
\hline$\bullet \quad$ Illiterate & 44 & 73.3 \\
\hline - $\quad$ Primary & 6 & 10 \\
\hline - $\quad$ Secondary & 10 & 16.7 \\
\hline \multicolumn{3}{|l|}{ 5-Occupation: } \\
\hline$\bullet \quad$ House wife & 55 & 91.7 \\
\hline$\bullet \quad$ Worker & 5 & 8.3 \\
\hline \multicolumn{3}{|l|}{ 6- Residence: } \\
\hline • Urban & 5 & 8.3 \\
\hline - $\quad$ Rural & 55 & 91.7 \\
\hline
\end{tabular}


Table (2): Distribution of patients according to dietary habits $(n=60)$.

\begin{tabular}{|c|c|c|}
\hline Items & $\mathrm{n}=(\mathbf{6 0})$ & $\%$ \\
\hline \multicolumn{3}{|l|}{ Use of tea and coffee } \\
\hline- Yes & 54 & 90.0 \\
\hline$-\mathrm{No}$ & 6 & 10.0 \\
\hline \multicolumn{3}{|l|}{ Use of alcohol } \\
\hline- No & 60 & 100 \\
\hline \multicolumn{3}{|l|}{ Smoking } \\
\hline$-\mathrm{No}$ & 60 & 100 \\
\hline \multicolumn{3}{|l|}{ Types of diet } \\
\hline - Plant based diet(vegetarian) & 2 & 3.3 \\
\hline - Plant based diet(semi- vegetarian) & 54 & 90.0 \\
\hline - Mainly meat-based diet & 4 & 6.7 \\
\hline
\end{tabular}

Table (3): Nutritional status ssessment for patients undergoing chemotherapy $(\mathrm{n}=60)$.

\begin{tabular}{|l|c|c|}
\hline \multirow{2}{*}{ Items } & \multicolumn{2}{c|}{ Mini Nutritional Assessment } \\
\cline { 2 - 3 } & $\mathbf{N = 6 0}$ & \% \\
\hline - $\quad$ Normal nutrition status & 53 & 88.3 \\
\hline - $\quad$ At risk of malnutrition & 7 & 11.7 \\
\hline
\end{tabular}

Table (4): Distribution of patients according to chemotherapy knowledge, attitude and practice $(\mathrm{n}=60)$.

\begin{tabular}{|l|c|c|}
\hline \multicolumn{1}{|c|}{ Variables } & No & $\%$ \\
\hline 1. Knowledge & & \\
\hline - Satisfy & 24 & $40 \%$ \\
\hline - Unsatisfied & 36 & $60 \%$ \\
\hline 2. Attitude & & $95 \%$ \\
\hline - Positive attitude & 57 & $5 \%$ \\
\hline - Negative attitude & 3 & $13.33 \%$ \\
\hline 3. Practice & & $86.66 \%$ \\
\hline - Good & 8 & 100 \\
\hline - Poor & 52 & \\
\hline - Total & 60 & \\
\hline
\end{tabular}

Table (5): Distribution of the studied sample regarding family history, performance status, stage and duration of cancer $(n=60)$.

\begin{tabular}{|l|c|c|}
\hline \multicolumn{1}{|c|}{ Items } & N. \\
\hline Performance status The Eastern Cooperative Oncology Group (ECOG): \\
\hline Score 0 Full active & 0 & 0 \\
\hline Score 1 Restricted in physical activity & 52 & 86.7 \\
\hline Score 2 Ambulatory and capable of all self-care & 5 & 8.3 \\
\hline Score 3 Capable of only limited self-care & 3 & 0 \\
\hline Score 4 Completely disabled & 0 & 0 \\
\hline Score 5 Dead & 0 & $8.3 \%$ \\
\hline 1. Stage of cancer & & 40 \\
\hline Stage 1 & 5 & 50 \\
\hline Stage 2 & 24 & 1.7 \\
\hline Stage 3 & 30 & 85 \\
\hline Stage 4 & 1 & 15 \\
\hline 2. Duration of cancer & & \\
\hline 1-5years & 51 & \\
\hline 6-10years & 9 & \\
\hline
\end{tabular}


Table (1): Mentioned that, less than half of patients $(36.7 \%)$ had age of $41-49$ years, all of them (100\%) were females, vast majority of them $(96.7 \%)$ were married, and most of them $(73.3 \%)$ were illiterate. Concerning of occupation most of them (91.7\%) not working, and (91.7) of them living in rural area.

Table (2): This table demonstrated that; nearly all the patients neither drinking tea, coffee, nor alcohol, also were not smoking, concerning type of diet $(90 \%)$ were consuming plant based diet (semi-vegetarian)

Table (3): This table should that the majority of samples $(88.3 \%)$ were at normal nutrition status

Table (4): This table explained that; more than two thirds of samples were unsatisfied level of knowledge $(60 \%)$. The majority of studied sample were positive attitude and poor practice $(95 \%, 86.6 \%)$ respectively.

Table (5): This explained that; according to family history of cancer most of the patients $(88.3 \%)$ had no family history. Regarding performance status (ECOG) the majority of them $(86.7 \%)$ regarding physical activity were restricted and $50 \%$ were had cancer in grade 3 . As regard duration of disease most of them $(85.0 \%)$ were complaining of cancer from 15 years

\section{Discussion}

Most patients diagnosed with cancer will receive chemotherapy, and patients undergoing medication experience and large number of symptoms, including fatigue, pain, dyspnea, nausea, loss of appetite, and unintentional weight change. These symptoms can negatively impact one's ability to complete treatment as well as one's quality of life during and after treatment (Coa et al., 2015).

A surprisingly high number of patients undergoing cancer treatment report distortions to their sense of taste or smell. Cancer patients also report early satiety and food aversions as a result of treatment. Dietary interventions, such as dietary counseling, flavor enhancement, oral supplementation, or tube feeding, have been found to lessen weight loss and improve health status (Barbera et al., 2010)

Most of the research exploring the relationship between diet and health status/quality of life during cancer treatment has focused on specific subgroups, such as patients with advanced cancer or patients with head and neck cancers, and include smaller sample sizes. However, a recent study of 1453 outpatients suggested that a broader range of patients are at nutritional risk during cancer treatment. Despite this, nutritional screenings are not routinely conducted with cancer patients. (Capuano, et al., 2008)

In my study the majority of patients were married. According to (Texeira \& da Graca, 2014) in their study "Marital Status and Survival in Patients with
Cancer", single patients were at significantly higher risk of presentation with metastatic cancer, under treatment, and death resulting from their cancer.

The present study presented that the majority of the patients in the age range of 41 - 49 yrs. This disagree with ( Jarvandi, 2016) who mentioned in his study, that two thirds of all newly diagnosed female breast cancer patients are in the age 55 years and older and this can be attributed to the postmenopausal period of life. According to (Coa, et al., 2015) younger patients were more likely than older patients to report chemosensory alterations, which may be attributable to olfactory and gustatory functions diminishing with age.

This study found that nearly all patients drink tea, coffee, not use alcohol, smokers from opinion of the researcher this is related to all the sample was female in rural area don't smok or drink alcohol. Concerning type of diet they were having that plant based diet (semi-vegetarian), and this suggests good dietary habits. This proved the result that one third of the sample were at astandard level of weight .This confirms the opinion of (Scholz et al., 2015) who concluded that dietary habits at the time of diagnosis may affect prognosis, at least for patients with estrogen receptor -rich breast cancers.

According to (James et al., 2015) the pattern of body-fat distribution also influences the risk of breast cancer.

Historically, weight loss in cancer patients has been the concern because of the association with poor prognosis (Fearon, et al., 2011) . particularly breast cancer patients were unique in that they were more likely to gain weight following treatment than loss weight. There is an adverse relationship between weight change and prognosis for breast cancer patients (Kroenke, 2005) and consequently patients likely to gain weight need nutritional advice on how to avoid gaining weight. This highlights a shift in the relationship between cancer and weight.

The present study explained that more than half of patients had poor knowledge about chemotherapy, and therefore poor practice, and it increased post guidelines application, this corresponds with (O'Brien et al., 2013) who explained in their Investigation Helping Patients Make educated Choices: An Randomized Trial ofa choice support for adjuvant chemotherapy on Lymph Node-Negative Breast Cancer, that those patients received medical consultation alone have poor learning Furthermore. Fulfillment compared for the individuals which including the choice board wills their medical consultation alone have poor knowledge and satisfaction compared with those which adding the decision board to their medical consultation. 
Finally; the result suggests that nutritional intervention should be initiated before chemo radiotherapy, and it needs to be continued after treatment completion.

\section{Conclusion}

About two third of the sample level of knowledge were unsatisfactory. The majority of the sample was positive attitude and had poor practice respectively.

\section{Recommendations}

- Nutritional support, advice and guidelines by dietician of the nutritional regimen should be undertaken for patients undergoing chemotherapy to prevent occurrence of complications and improve outcomes.

- Formulate nutritional assessment form or sheet to be suitable for Egyptian patients undergoing chemotherapy in the hospitals.

- All laboratory investigations and measurements, which are essential for assessing nutritional status of patients undergoing chemotherapy should be done

- Identify how to calculate ideal body weight for those patients, to identify who are at risk for developing underweight or overweight and record results.

- Replication of the study on a larger probability sample acquired from different geographical areas in Egypt to figure out the main aspects of this problem.

\section{References}

4. Barbera L., Seow H., Howell D., Sutradhar R., \& Earle (2010): Symptom burden and performance status in a population-based cohort of ambulatory cancer patients. Cancer. Pp. 57675776.

5. Capuano G., Grosso A., Gentile P., Battista M., \& Bianciardi F., (2008): Influence of weight loss on outcomes in patients with head and neck cancer undergoing concomitant chemoradiotherapy, Head\& Neck, .Pp. 503-508.

6. Coa, K., Epstein, J., Ettinger, D., Jatoi, A., McManus, K., Platek, M., \&Moskowitz, B., (2015): The impact of cancer treatment on the diets and food preferences of patients receiving outpatient treatment. Nutrition and cancer, 67(2),Pp. 339-353.

7. Fearon K., Strasser F., Anker S., Bosaeus I., \& Bruera E., (2011): Definition and classification of cancer cachexia: an international consensus. The Lancet Oncology,. Pp. 489:495.

8. James, F., Wootton, S., Jackson, A., Wiseman, M., Copson, E., \&Cutress, R., (2015): Obesity in breast cancer-what is the risk factor?. European journal of cancer, 51(6), Pp.705-720.

9. Jarvandi, S., Pérez, M., Schootman, M., \& Jeffe, D., (2016): Pre-Existing Diabetes in Early Stage Breast Cancer Patients is Associated with Lack of Improvement in Quality of Life 2 Years After Diagnosis. International journal of behavioral medicine, 23(6),Pp. 722-729.

10. Jcoyle, Thaiheimer, (2013): plus the top five foods that can lower LDL Cholesterol pp(405-420

11. Jemal A., Bray F., Center M., Ferlay J., Ward E., \& Forman D., (2011): "Global cancer statistics voume 61 (2).Pp. 9-90.

12. Koyabu N., \& Ohtani H., (2012): "Inhibition of Pglycoprote by flavonoid derivatives in adriamycin-resistant human myelogenous leukemia cells," Cancer Letters, vol. 177, no. 1.Pp. 89-93.

13. Kroenke C., Chen W., Rosner B., \& Holmes M., (2005): Weight, weight gain, and survival after breast cancer diagnosis, Journal of Clinical Oncology, .Pp.1370:1378.

14. Mariani L., Bozzetti F., ( 2012): The nutritional risk in oncology: a study of 1,453 cancer outpatients. Supportive Care in . Pp. 1919-1928.

15. Morello M., Paccagnella A., Maria C., Baruffi C., Marcon M., Gava A., Baggio V., Lamon S., Babare R., Rosti G., Giometto M., BoscoloRizzo B., Kiwanuka E., Tessarin M., Caregaro L., \& Marchiori C., (2010): Early nutritional intervention improves treatment tolerance and outcomes in head and neck cancer patients undergoing concurrent chemo radiotherapy, Supportive Care in Cancer, Volume 18, Issue 7, p.p. 837:845.

16. Nafissi \& Raychaudhur, (2012): Cancer survivorship issues: life after treatment and implications for an aging population. Journal of Clinical Oncology, 32(24),Pp. 2662-2668

17. O'Brien, M., Ellis, P., Whelan, T., Charles, C., Gafni, A., Lovrics, \& Hodgson, N., (2013): Physicianrelated facilitators and barriers to patient involvement in treatment decision making in early stage breast cancer: perspectives of physicians and patients. Health Expectations, 16(4), 373-384.

18. Pantavos, A., Ruiter, R., Feskens, E., Keyser, C., Hofman, A., Stricker, B., \& Kieftede Jong, J., (2015): Total dietary antioxidant capacity, individual antioxidant intake and breast cancer risk: The Rotterdam study. International journal of cancer, 136(9), Pp.2178-2186.

19. Scholz, C., Andergassen, U., Hepp, P., Schindlbeck, C., Friedl, T., Harbeck, N., \& Rack, B., (2015): Obesity as an independent risk factor for decreased survival in node-positive 
high-risk breast cancer. Breast cancer research and treatment, 151(3),Pp. 569-576

20. Texeira, R., \& da Graca Pereira, M., (2014): Mind-Body Health Promotion and Women's Cancers. The Praeger Handbook on Women's Cancers: Personal and Psychosocial Insights: Personal and Psychosocial Insights, 151. 